\title{
Cardiotoxicity by Anthracycline Regimen Chemotherapy Prolonged T Peak to T End Interval
}

\author{
Mohammad Iqbal $^{\text {a, }}$, Viky Victory ${ }^{\mathrm{a}}$, Astri Astutia ${ }^{\mathrm{a}}$, Mega Febrianora ${ }^{\mathrm{a}}$, Giky Karwiky ${ }^{\mathrm{a}}$, \\ Chaerul Achmad ${ }^{\text {a }}$, Mohammad Rizki Akbar ${ }^{\mathrm{a}}$
}

\begin{abstract}
Background: Myocardial necrosis may occur due to anthracycline (doxorubicin/adriamycin) chemotherapy usage. Furthermore, myocardial necrosis can affect the heterogeneity of heart conduction system and lead to repolarization abnormalities. The aim of this study was to investigate the effect of cardiotoxicity caused by anthracycline to repolarization abnormalities measured by $\mathrm{T}$ peak to $\mathrm{T}$ end $(\mathrm{TpTe})$ interval.
\end{abstract}

Methods: This was a single center prospective cohort study with linear regression from October 2018 to May 2019. The subjects of the study were breast cancer patients after completing administration of chemotherapy with fluorouracil, adriamycin and cyclophosphamide (FAC) regimen (containing anthracycline) for 6 months. Myocardial necrosis was assessed by high sensitive (hs)-troponin $\mathrm{I}$, and the heterogeneity of repolarization was measured by TpTe interval.

Results: This study involved 25 breast cancer patients after chemotherapy in the 6-month FAC regimen. The mean age is $46 \pm 7$ years, and the cumulative dose of anthracycline is $591 \pm 52 \mathrm{mg} / \mathrm{m}^{2}$. The mean level of hs-troponin I is $90.5 \pm 44.7 \mathrm{ng} / \mathrm{L}$ and the TpTe interval is $108.2 \pm 10 \mathrm{~ms}$. The results of linear regression analysis showed a positive correlation between hs-troponin I and TpTe interval (r: 0.421, P: 0.036) after controlling for one confounding variable (cumulative dose of anthracycline).

Conclusions: Cardiotoxicity caused by accumulative dose of anthracycline may lead to myocardial necrosis which was shown by elevated hs-troponin I levels. This process may lead to heterogeneity conduction system that affect the repolarization phase of cardiac cycle which was shown by increased TpTe interval.

Keywords: Cardiotoxicity; Hs-troponin I; TpTe interval

Manuscript submitted May 29, 2020, accepted June 12, 2020

Published online August 1, 2020

aDepartment of Cardiology and Vascular Medicine, Hasan Sadikin General Hospital, Jalan Eyckman 38, Bandung 40161, Indonesia

${ }^{b}$ Corresponding Author: Mohammad Iqbal, Department of Cardiology and Vascular Medicine, Hasan Sadikin General Hospital, Jalan Eyckman 38, Bandung 40161, Indonesia. Email: mohammadiqbal178@gmail.com

doi: https://doi.org/10.14740/cr1052

\section{Introduction}

Chemotherapy using FAC regimen which consists of 5-fluorouracil (5-FU), adriamycin (doxorubicin (DOX)/anthracycline), and cyclophosphamide (CYA) is a therapy that is widely used in the world, and is also a therapeutic standard in cancer treatment. However, the downside of using FAC chemotherapy is cardiotoxicity mainly due to anthracycline. The spectrum of cardiotoxicity varies widely such as pericardial effusion, heart failure, myocardial infarct, and arrhythmia $[1,2]$.

Cardiotoxicity can cause myocardial damage which affect conduction system and lead to repolarization abnormality. Damage to the myocardium can be assessed by increased troponin level. Test of high sensitive-troponin I (hs-troponin I) could be used to detect a very low level of troponin, thus can evaluate very minor myocardial injury before clinical myocardial dysfunction occurs [3-5]. However, damage to endocardium and myocardium caused by chemotherapy leads to repolarization disruption across cardiac myocytes [6]. T peak to $\mathrm{T}$ end $(\mathrm{TpTe})$ interval is one of the most frequently used electrocardiogram (ECG) markers to evaluate repolarization heterogeneity representing global and transmural dispersion of repolarization [7]. This study was conducted to evaluate the effect of cardiotoxicity caused by cumulative doses of anthracycline to ventricular repolarization heterogeneity represented by TpTe interval.

\section{Materials and Methods}

\section{Study design and population}

Our study was a prospective cohort study. Subjects were breast cancer patients older than 18 years old who were part of Breast Cancer Registry at Hasan Sadikin Hospital starting from October 2018. Subjects who had sufficient data including hs-troponin I, renal function, baseline ECG before initiating chemotherapy and completed six cycles of FAC were asked to do a follow-up visit and underwent subsequent ECG and hstroponin I examination. The exclusion criteria were positive hs-troponin I before chemotherapy, heart failure, pulmonary embolism, renal failure, acute coronary syndrome, stroke, or abnormal TpTe on baseline ECG. A total of 25 patients were included after applying the exclusion criteria. 


\section{Electrocardiography and hs-troponin I}

Cardiotoxicity or myocardial necrosis was measured by hstroponin I. A standard 12-lead surface ECG was obtained at $25 \mathrm{~mm} / \mathrm{s}$. The TpTe interval is measured from the top of the $\mathrm{T}$ wave to the end of the $\mathrm{T}$ wave in the precordial leads on the 12-lead ECG record. The end of the T wave is determined by the tangential method, which is defined as the intersection of the isoelectric line with the tangent corresponding to the $\mathrm{T}$ wave slope [8].

Lead with a $T$ wave amplitude smaller than $1 \mathrm{~mm}$ or not finding an obvious $\mathrm{T}$ wave should not be taken into account in the measurement. $\mathrm{T}$ waves with negative, biphasic, and betadeflection should be excluded because the TpTe interval is difficult to determine. In the $\mathrm{T}$ wave followed by the $\mathrm{U}$ wave, the determination of the tangent line is measured based on the slope of the nadir between the $T$ wave and wave $U$.

To the best of our knowledge, there is no standard for determining the leads used in the TpTe interval measurement. Some previous publications used tangential methods in various different leads, namely the TpTe interval in leads V2, V5, $\mathrm{II}$, averages at V1 - V6, averages at V4 - V6, maximum intervals of V1 - V6, and intervals maximum of all leads. Other studies used automatic measurements using the GE Healthcare 12SL/QT Guard Plus analysis program (method $12 \mathrm{SL}$ ). Rosenthal's study compared all the measurement methods in 305 cardiomyopathy patients and found that the majority of these measures were useful for the risk of ventricular arrhythmias (ventricular tachycardia/fibrillation) and mortality, but manual measurements with the tangential method in V2 and automatic measurement by the $12 \mathrm{SL}$ method are the most clinically useful methods [8].

QTc interval was measured by Bazzet formula. All the measurement was done in all 12-lead ECG by an experienced cardiologist, and the longest measured interval was used for analysis. Hs-troponin I was quantified using Vidas Ultra, Biomeurieux. The 99th percentile reference value was $100 \mathrm{ng} / \mathrm{L}$. All values above this reference value were considered positive.

\section{Statistical analysis}

Continuous variables were expressed as means \pm standard deviations (SD) or median (range) if data were not distributed normally. The Saphiro-Wilk test was used to assess the normality of data. Categorical data are summarized as frequencies and percentages. Pearson correlation or Spearman as an alternative was used to determine the correlation coefficient. Correlation coefficient was deemed very low, low, moderate, strong, very strong according to $\mathrm{r}$ value $(0-0.199,0.2-0.399$, $0.4-0.599,0.6-0.799$, and $0.8-1.0$, respectively). Statistical analyses were performed with STATA, version 24.0 (STATA Corp LCC, Texas, USA).

\section{Ethical standards}

The study protocol was reviewed and approved by the Re-
Table 1. Baseline Characteristics

\begin{tabular}{ll}
\hline Characteristics & $\mathbf{N}=\mathbf{2 5}$ \\
\hline $\begin{array}{l}\text { Age }(\text { years }), \text { mean } \pm \mathrm{SD} \\
\text { BMI }\left(\mathrm{kg} / \mathrm{m}^{2}\right), \text { mean } \pm \mathrm{SD}\end{array}$ & $46 \pm 7$ \\
$\begin{array}{l}\text { Anthracycline cumulative dose }\left(\mathrm{mg} / \mathrm{m}^{2}\right), \text { mean } \pm \mathrm{SD} \\
\text { Comorbid, frequency, } \%\end{array}$ & $591 \pm 52$ \\
$\quad$ Hypertension & $7(28 \%)$ \\
$\quad$ Diabetes mellitus & $4(16 \%)$ \\
$\quad$ Dyslipidemia & $2(8 \%)$ \\
Medication, frequency $\%$ & \\
$\quad \begin{array}{l}\text { ACEI/ARB } \\
\quad \text { Calcium channel blocker }\end{array}$ & $5(20 \%)$ \\
QTc interval (ms), mean \pm SD & $2(8 \%)$ \\
LVEF $(\%)$, mean \pm SD & $462.5 \pm 21.9$ \\
\hline
\end{tabular}

BMI: body mass index; SD: standard deviation; ACEI: angiotensin-converting enzyme inhibitor; ARB: angiotensin II receptor blocker; LVEF: left ventricular ejection fraction.

search Ethics Committee, Faculty of Medicine, Universitas Padjadjaran, Bandung; and the study was conducted in compliance with the ethical standards of the responsible institution on human subjects as well as with the Helsinki Declaration.

\section{Results}

\section{Patient characteristics}

We enrolled 25 patients with average age of $46 \pm 7$ years and anthracycline cumulative dose of $591 \pm 52 \mathrm{mg} / \mathrm{m}^{2}$. The majority of population did not have traditional cardiovascular risk factor. The hypertension is the most common comorbidity in only seven subjects (28\%). The medications of angiotensinconverting enzyme inhibitor (ACEI)/angiotensin II receptor blocker (ARB) (ramipril and candesartan) and calcium channel blocker (amlodipine) were used among patient with hypertension. The baseline of left ventricular ejection fraction (LVEF, \%) was $67.4 \pm 4.7 \%$ (Table 1).

\section{TpTe interval and hs-troponin I}

Both values of TpTe interval and hs-troponin I increased considerably after six cycles of chemotherapy using FAC regimen. Hs-troponin I value before chemotherapy was $4.5 \mathrm{ng} / \mathrm{L}$, and after six cycles of chemotherapy it was $90.5 \mathrm{ng} / \mathrm{L}$. TpTe interval was $73.9 \mathrm{~ms}$ before chemotherapy and after six cycles of chemotherapy it was $108.2 \mathrm{~ms}$. Correlation was considered moderate (r: 0.421, P: 0.018), therefore we concluded that the increase of hs-troponin I would be accompanied by the increase of TpTe interval (Table 2, Fig. 1). However, during the period of chemotherapy, there was no patient who had symp- 
Table 2. Hs-Troponin I and TpTe Values

\begin{tabular}{lllll}
\hline Variable & Baseline & After chemotherapy & Correlation coefficient & P value \\
\hline Hs-troponin I (ng/L) & $4.5(1.5-15)$ & $90.5 \pm 44.7$ & 0.421 & 0.018 \\
TpTe interval (ms) & $73.9 \pm 59.8$ & $108.2 \pm 10$ & & \\
\hline
\end{tabular}

Hs-troponin I: high sensitive troponin I; TpTe: T peak to T end.

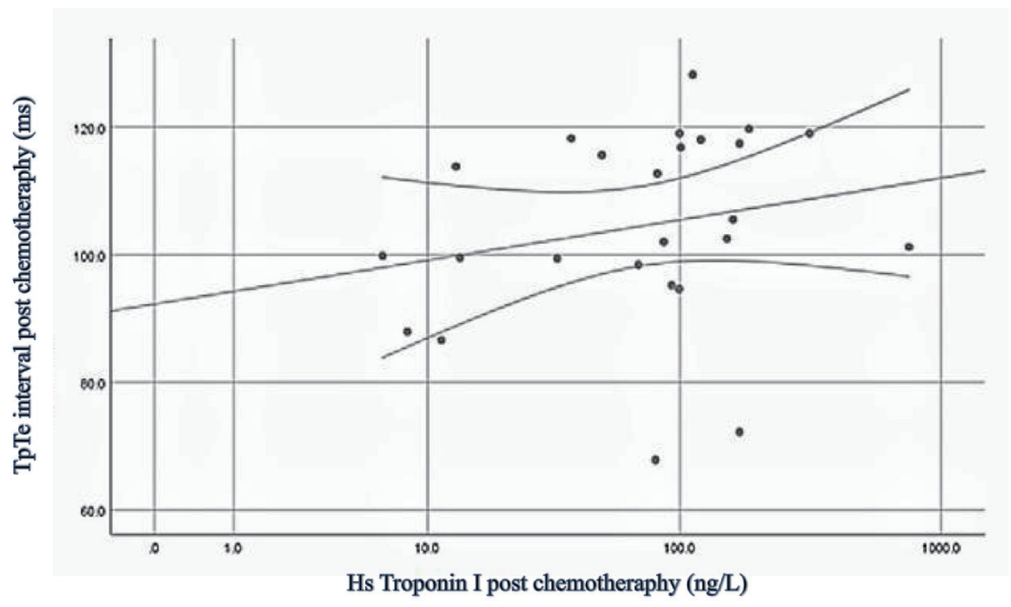

Figure 1. Scatter plot of hs-troponin I to TpTe interval. Hs: high sensitive; TpTe: T peak to T end.

toms related to ventricular arrhythmia (such as palpitation, near syncope or syncope) which was related to increased TpTe interval, and no documented ECG of ventricular arrhythmia during visitation in outpatient clinic.

Table 3 shows univariable logistic regression analysis. Univariable analysis confirmed the association between cumulative anthracycline dose and TpTe interval but not age, body mass index (BMI), or traditional risk factors. We did further analysis using multivariable logistic regression analysis (Table 4) to examine the effect of cumulative anthracycline dose upon the correlation between hs-troponin I and TpTe interval. Analysis showed significant moderate correlation between hs-troponin I and TpTe (r: 0.421, P: 0.036). Hstroponin I has B coefficient of 0.094 which is translated to that every $1 \mathrm{ng} / \mathrm{L}$ increase of hs-troponin I will be accompanied by an increase of TpTe around $0.094 \mathrm{~ms}$. Of note, there were no patient consumed of drugs that might effect to repolarization during chemotherapy period. The difference of QTc (ms) before $(462.5 \pm 21.9)$ and after $(476.9 \pm 36.2)$ did not show statistical significance $(\mathrm{P}=0.58)$, neither did the difference of LVEF (\%) before $(67.4 \pm 4.7)$ and after chemotherapy (65.8 $\pm 6.9)(\mathrm{P}=0.15)$.

\section{Discussion}

FAC regimens are widely used for the treatment of breast cancer today. FAC consists of 5-FU, anthracycline (DOX/ adriamycin) and CYA. Of the three drugs, Anthracycline is the drug that most often causes cardiotoxicity. Anthracycline (DOX) was the most cytotoxic drug, followed by 5-FU and lastly CYA in both cytotoxicity assays; even in the presence of higher concentrations of 5-FU and CYA $(50 \mu \mathrm{M} 5-\mathrm{FU}+1 \mu \mathrm{M}$ DOX $+50 \mu \mathrm{M}$ CYA), $1 \mu \mathrm{M}$ DOX was still a determinant for the cardiotoxicity observed in the cytotoxicity assays, phase contrast morphological evaluation, and mitochondrial potential depolarization evaluation [9].

Anthracycline (DOX) would induce oxidative stress and

Table 3. Univariate Analysis of Confounding Factors

\begin{tabular}{lll}
\hline \multirow{2}{*}{ Variable } & \multicolumn{2}{c}{ T peak to T end } \\
\cline { 2 - 3 } & Correlation coefficient & P value \\
\hline Age (years) & -0.169 & 0.420 \\
BMI $\left(\mathrm{kg} / \mathrm{m}^{2}\right)$ & -0.180 & 0.389 \\
Cumulative anthracycline dose & 0.407 & 0.044 \\
Hypertension & -0.191 & 0.361 \\
Diabetes mellitus & -0.367 & 0.071 \\
\hline
\end{tabular}

BMI: body mass index. 
Table 4. Multivariate Analysis

\begin{tabular}{|c|c|c|c|c|c|}
\hline \multirow{2}{*}{ Variable } & \multicolumn{2}{|c|}{ Unstandardized coefficients } & \multirow{2}{*}{$\begin{array}{l}\text { Standardized coefficients } \\
\text { Beta }\end{array}$} & \multirow{2}{*}{ P value } & \multirow{2}{*}{$\mathbf{R}^{2}$} \\
\hline & B & SE & & & \\
\hline Constants & 57.528 & 20.121 & & 0.009 & 0.318 \\
\hline Cumulative anthracycline dose & 0.072 & 0.034 & 0.377 & 0.044 & \\
\hline Constants & 99.642 & 4.274 & & $<0.001$ & 0.177 \\
\hline
\end{tabular}

Hs-troponin I: high sensitive troponin I; SE: standard error.

inhibit topoisomerase II in patients causing myocardial damage and the subsequent increase of hs-troponin I $[10,11]$. This myocardial injury would also cause heterogeneity of myocardial repolarization leading to an increased risk of ventricular arrhythmia and sudden cardiac death $[12,13]$. The increase of hs-troponin I after six cycles of chemotherapy in our study shows considerable myocardial injury after repeated exposure to anthracycline. Sawaya et al stated that hs-troponin I value of more than $15 \mathrm{ng} / \mathrm{L}$ after chemotherapy could predict cardiotoxicity and reduction of LVEF within 6 months [14].

Average TpTe interval after chemotherapy in our study was $108.2 \pm 10 \mathrm{~ms}$. Benatar et al showed an increase of TpTe 6 months after chemotherapy with anthracycline cumulative dose between $185-480 \mathrm{mg} / \mathrm{m}^{2}$ compared to control $(109 \pm 17$ ms vs. $92 \pm 14 \mathrm{~ms}$ ) [15], even though their study population only includes children with average age of 13 years old with lower cumulative dose compared to our study. Several studies have shown that TpTe could predict ventricular arrhythmia and sudden cardiac death, even though normal reference value for TpTe has not been agreed upon $[16,17]$. Aro et al found a TpTe value of more than $89 \mathrm{~ms}$ to be significantly associated with sudden cardiac death [17]. Meta-analysis by Tse et al concluded that TpTe has different cut-off in predicting ventricular arrhythmia depending on the underlying disease. Value of $>$ $113.6 \mathrm{~ms}$ in normal population, $>95.8 \mathrm{~ms}$ in Brugada syndrome, $>106.3 \mathrm{~ms}$ in heart failure, $>109.6 \mathrm{~ms}$ in ischemic heart disease were the cut-off considered to be important [18]. The average TpTe value that has strong association with ventricular arrhythmia was $103.3 \pm 17.4 \mathrm{~ms}$, thus subjects in our study already had an increased risk of ventricular arrhythmia and sudden cardiac death.

Our univariate analysis showed cumulative anthracycline dose as a confounding factor for the increase of TpTe. Some studies have shown that LV systolic dysfunction occurs at cumulative dose $>550 \mathrm{mg} / \mathrm{m}^{2}$ while others found heart failure incidence of 3-5\% in cumulative dose of $400 \mathrm{mg} / \mathrm{m}^{2}$ [19-21]. The average cumulative dose in our study was $591 \pm 52 \mathrm{mg} /$ $\mathrm{m}^{2}$. It was considered high in accordance to previous studies. However, our study showed that the LVEF after chemotherapy tend to be lower compared to baseline $(65.8 \pm 6.9$ vs. $67.4 \pm$ $4.7 ; \mathrm{P}=0.15$ ) but difference not statistically significant. Some factors may affect this result. Previous study showed that older age may increase the risk of LV systolic dysfunction due to chemotherapy [19-21]. The mean age in our study showed that our population is younger compared the previous study [20,
21]. Furthermore, the LV systolic dysfunction still may occur up to 231 days after last chemotherapy [19]. Therefore, the risk of significant LV systolic dysfunction in our study still needs to be determined in long-term follow-up.

Our study found significant moderate correlation between hs-troponin I and TpTe (r: 0.421, P: 0.036). We conclude that there was an association between myocardial injury (marked by an increase of hs-troponin I more than $15 \mathrm{ng} / \mathrm{L}$ compared to baseline) and heterogeneity of myocardial repolarization (marked by high value of TpTe post chemotherapy). Rajvanshi et al [22] also found an association between repolarization heterogeneity (represented by QT interval) and myocardial injury quantified by troponin I in patients with acute coronary syndrome.

Hs-troponin I evaluation could be useful for detecting early myocardial damage after chemotherapy and is associated with the incidence of ventricular arrhythmia in breast cancer patients receiving anthracycline. This was the first study that analyzed association between hs-troponin I and TpTe interval in breast cancer patients receiving chemotherapy containing anthracycline.

\section{Limitations}

This study has several limitations. First, we did not evaluate serial TpTe and hs-troponin I after each cycle of chemotherapy; therefore we could not evaluate the fluctuation of these parameters between each cycle. Second, there is no standard for determining the leads used in the TpTe interval measurement. Therefore, we could not speculate whether another technique for measurement of TpTE will show comparable result compared to our results. Third, we did not perform Holter or longer monitoring to detect ventricular arrhythmia that may relate to prolong TpTe. Even though all the patients had no symptoms of syncope, near syncope, palpitation neither documented ventricular arrhythmia on ECG during outpatient clinic, subclinical arrhythmia due to non-sustained torsade de pointes or other ventricular arrhythmia may be potentially undetected during follow-up. Therefore, continuous longer monitoring was needed to confirm it. Fourth, we did not examine long-term serial echocardiography after chemotherapy. Even though no significant difference of LV systolic dysfunction before and after chemotherapy was found, some of LV systolic dysfunction still may occur later. 


\section{Conclusions}

Cardiotoxicity caused by accumulative dose of anthracycline may lead to myocardial necrosis which was shown by elevated hs-troponin I levels. This process may lead to heterogeneity of conduction system that affects the repolarization phase of cardiac cycle which was shown by increased TpTe interval.

\section{Acknowledgments}

None to declare.

\section{Financial Disclosure}

This research received no specific grant from any funding agency, commercial or not-for-profit sectors.

\section{Conflict of Interest}

All authors declare no conflict of interest related to this study.

\section{Informed Consent}

Informed consents were obtained from all patients.

\section{Author Contributions}

MI made conception and design of the study, implemented the study, analysed and interpreted the study results, drafted the manuscript and revised it. VV, AA, MF, GK, CA, and MRA contributed to the design of study, analysis, and interpretation of study data.

\section{Data Availability}

Any inquiries regarding supporting data availability of this study should be directed to the corresponding author MI.

\section{Abbreviations}

TpTe: T peak to T end; FAC: fluorouracil, adriamycin, and cyclophosphamide; ECG: electrocardiogram

\section{Reference}

1. Brana I, Tabernero J. Cardiotoxicity. Ann Oncol. 2010;21(Suppl 7):vii173-179.

2. Jain D, Ahmad T, Cairo M, Aronow W. Cardiotoxicity of cancer chemotherapy: identification, prevention and treatment. Ann Transl Med. 2017;5(17):348.

3. Cardinale D, Sandri MT, Martinoni A, Borghini E, Civelli M, Lamantia G, Cinieri S, et al. Myocardial injury revealed by plasma troponin I in breast cancer treated with high-dose chemotherapy. Ann Oncol. 2002;13(5):710715 .

4. Ky B, Putt M, Sawaya H, French B, Januzzi JL, Jr., Sebag IA, Plana JC, et al. Early increases in multiple biomarkers predict subsequent cardiotoxicity in patients with breast cancer treated with doxorubicin, taxanes, and trastuzumab. J Am Coll Cardiol. 2014;63(8):809-816.

5. Sandri MT, Cardinale D, Zorzino L, Passerini R, Lentati P, Martinoni A, Martinelli G, et al. Minor increases in plasma troponin I predict decreased left ventricular ejection fraction after high-dose chemotherapy. Clin Chem. 2003;49(2):248-252.

6. Buza V, Rajagopalan B, Curtis AB. Cancer TreatmentInduced Arrhythmias: Focus on Chemotherapy and Targeted Therapies. Circ Arrhythm Electrophysiol. 2017;10(8):e005443.

7. Panikkath R, Reinier K, Uy-Evanado A, Teodorescu C, Hattenhauer J, Mariani R, Gunson K, et al. Prolonged Tpeak-to-tend interval on the resting ECG is associated with increased risk of sudden cardiac death. Circ Arrhythm Electrophysiol. 2011;4(4):441-447.

8. Rosenthal TM, Masvidal D, Abi Samra FM, Bernard ML, Khatib S, Polin GM, Rogers PA, et al. Optimal method of measuring the T-peak to T-end interval for risk stratification in primary prevention. Europace. 2018;20(4):698705.

9. Pereira-Oliveira M, Reis-Mendes A, Carvalho F, Remiao F, Bastos ML, Costa VM. Doxorubicin is key for the cardiotoxicity of FAC (5-Fluorouracil + Adriamycin + Cyclophosphamide) combination in differentiated $\mathrm{H} 9 \mathrm{c} 2$ cells. Biomolecules. 2019;9(1).

10. Adamson PD, Hall P, Lang N, et al. Dynamic Changes in high sensitivity cardiac troponin I in response to anthracycline-based chemotheraphy: a pilot study for the cardiac care trial. Journal of the American College of Cardiology. 2018;71(11 Supplement):A701.

11. Jones M, O'Gorman P, Kelly C, Mahon N, Fitzgibbon MC. High-sensitive cardiac troponin-I facilitates timely detection of subclinical anthracycline-mediated cardiac injury. Ann Clin Biochem. 2017;54(1):149-157.

12. Suter TM, Ewer MS. Cancer drugs and the heart: importance and management. Eur Heart J. 2013;34(15):11021111.

13. Prenner SB, Shah SJ, Goldberger JJ, Sauer AJ. Repolarization heterogeneity: beyond the QT interval. J Am Heart Assoc. 2016;5(5).

14. Sawaya H, Sebag IA, Plana JC, Januzzi JL, Ky B, Cohen $\mathrm{V}$, Gosavi S, et al. Early detection and prediction of cardiotoxicity in chemotherapy-treated patients. Am J Cardiol. 2011;107(9):1375-1380.

15. Benatar A, Dewals W, Decraene T, Feenstra A. Ventricular repolarization intervals in children previously treated with anthracyclines. ARC Journal of Cardiology. 2017;3(2):6-15.

16. Porthan K, Viitasalo M, Toivonen L, Havulinna AS, Jula 
A, Tikkanen JT, Vaananen H, et al. Predictive value of electrocardiographic T-wave morphology parameters and T-wave peak to T-wave end interval for sudden cardiac death in the general population. Circ Arrhythm Electrophysiol. 2013;6(4):690-696.

17. Aro AL, Reinier K, Rusinaru C, Uy-Evanado A, Darouian N, Phan D, Mack WJ, et al. Electrical risk score beyond the left ventricular ejection fraction: prediction of sudden cardiac death in the Oregon Sudden Unexpected Death Study and the Atherosclerosis Risk in Communities Study. Eur Heart J. 2017;38(40):3017-3025.

18. Tse G, Gong M, Wong WT, Georgopoulos S, Letsas KP, Vassiliou VS, Chan YS, et al. The Tpeak - Tend interval as an electrocardiographic risk marker of arrhythmic and mortality outcomes: A systematic review and meta-analysis. Heart Rhythm. 2017;14(8):1131-1137.

19. Von Hoff DD, Layard MW, Basa P, Davis HL, Jr., Von Hoff AL, Rozencweig M, Muggia FM. Risk factors for doxorubicin-induced congestive heart failure. Ann Intern Med. 1979;91(5):710-717.

20. Swain SM, Whaley FS, Ewer MS. Congestive heart failure in patients treated with doxorubicin: a retrospective analysis of three trials. Cancer. 2003;97(11):2869-2879.

21. Zamorano JL, Lancellotti P, Rodriguez Munoz D, Aboyans V, Asteggiano R, Galderisi M, Habib G, et al. 2016 ESC Position Paper on cancer treatments and cardiovascular toxicity developed under the auspices of the ESC Committee for Practice Guidelines: the task force for cancer treatments and cardiovascular toxicity of the European Society of Cardiology (ESC). Eur Heart J. 2016;37(36):2768-2801.

22. Rajvanshi S, Nath R, Kumar M, Gupta A, Pandit N. Correlation of corrected QT interval with quantitative cardiac troponin-I levels and its prognostic role in Non-ST-elevation myocardial infarction. Int J Cardiol. 2017;240:5559. 